\title{
Developmental Dislocation of Hip Misdiagnosed as Traumatic Posterior Hip Dislocation
}

\author{
Chan Yong Park', Hyun Min Cho', Kyung Hag Lee ${ }^{2}$ \\ 'Department of Trauma Surgery, Pusan National University Hospital, Busan, Korea \\ ${ }^{2}$ Department of Orthopedic Surgery, National Medical Center, Seoul, Korea
}

A 47-year-old woman was presented to the emergency department. The limb of the patient was shortened, and her hip was mildly flexed, adducted, and internally rotated. Initially, the patient was misdiagnosed with a posterior hip dislocation. However, after careful history taking and radiologic evaluation, her final diagnosis was developmental hip dysplasia, which was a sequelae of poliomyelitis.

Key Words: Hip Dislocation; Hip Joint; Pelvic Bones; Joint Diseases; Acetabulum

(Trauma Image Proced 2016(1):7-9)

\section{CASE}

A 47-year-old woman was presented to our emergency department via another hospital. She fell from a 5-m height on a mountain. Immediate orotracheal intubation was performed owing to decreased mentality caused by acute subdural hematoma and subarachnoid hemorrhage. The medical records from the previous hospital noted failed manual reduction of the left hip. The external appearance of the patient's left lower limb was shortened, and the hip was mildly flexed, adducted, and internally rotated. The patient's family reported that she had a limping gait since early childhood due to poliomyelitis, and the status of her hip joint had never been diagnosed with a radiologic examination. Anteroposterior pelvic radiography showed a dysplastic acetabulum and high dislocation of the left femoral head and a scant sclerotic iliac portion that could be suspected as a false acetabulum (Fig. 1.). The three-dimensional computed

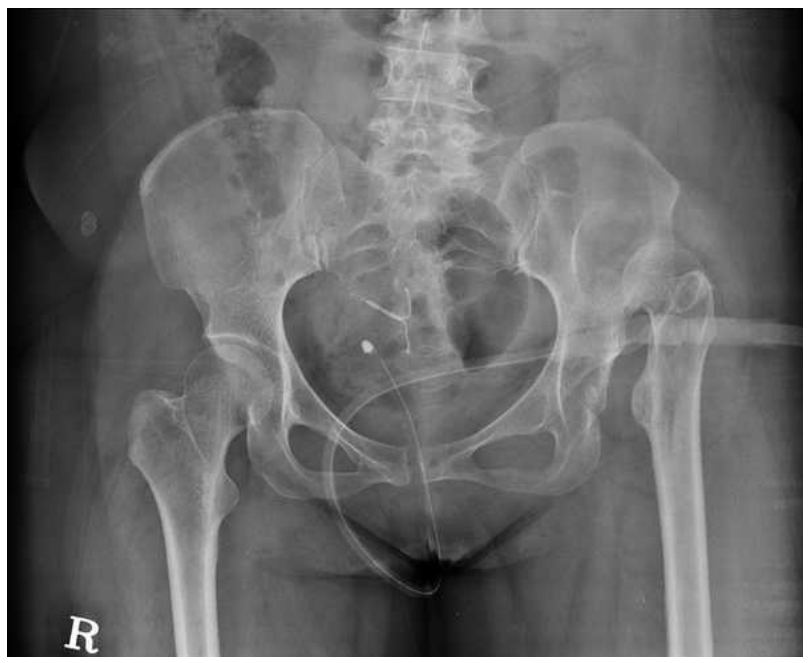

Fig. 1. Anteroposterior pelvic radiograph shows the dysplastic acetabulum and superior migration of the femoral head mimicking traumatic hip dislocation.

Received: May 24, 2016 Revised: June 15, 2016 Accepted: July 1, 2016

Correspondence to: Kyung-Hag Lee, Department of Orthopedic Surgery, National Medical Center, Euljiro 6-ga, Jung-gu, Seoul, Korea Tel: 82-2-2260-7199, Fax: 82-2-2276-2381, E-mail: hagine@hanmail.net

Copyright (c) 2016 Korean Association for Research, Procedures and Education on Trauma. All rights reserved.

@This is an open-access article distributed under the terms of the Creative Commons Attribution Non-Commercial License (http://creativecommons.org/ licenses/by-nc/4.0) which permits unrestricted noncommercial use, distribution, and reproduction in any medium, provided the original work is properly cited 

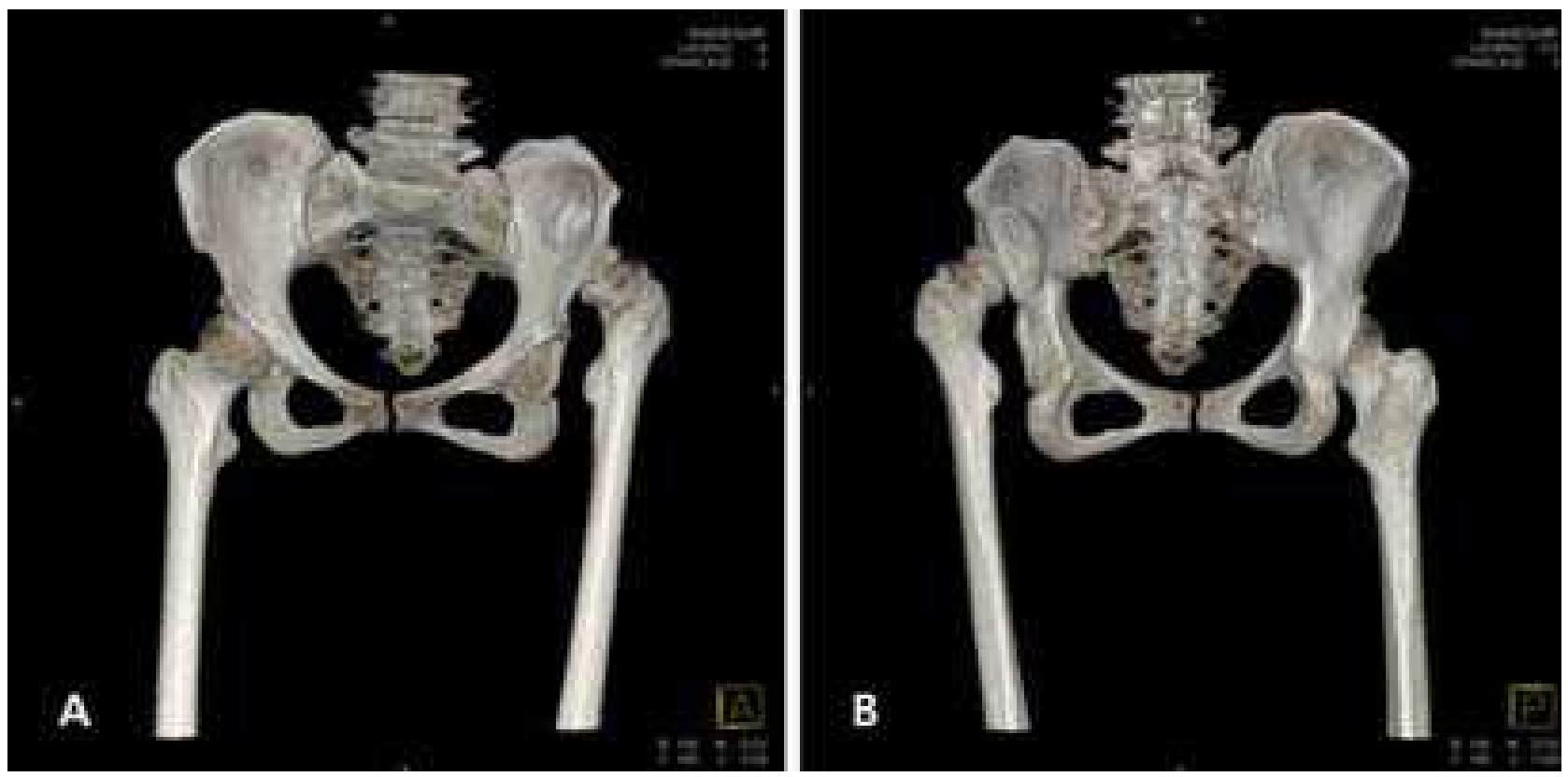

Fig. 2. Computed tomography scan of the pelvis shows articulation of the left femoral head with the false acetabulum in the ilium more clearly. (A) Anteroposterior view (B) posteroanterior view.

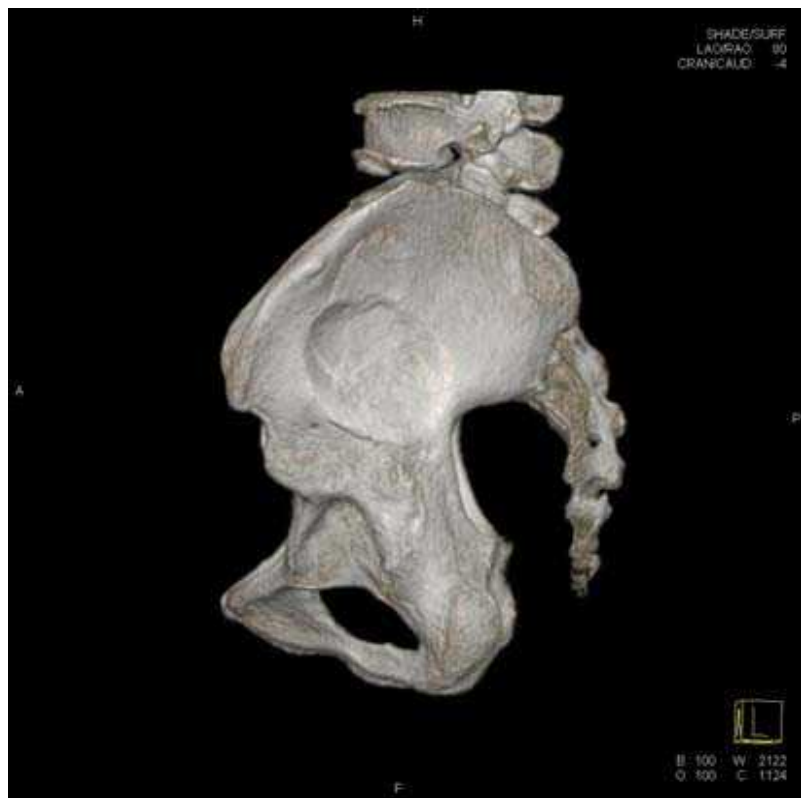

Fig. 3. Left lateral view of a computed tomography scan of the pelvis bone shows the round and deepened false acetabulum in the ilium.

tomography (CT) scan of the pelvis clearly shows the articulation of left femoral head with the false acetabulum in the ilium (Fig. 2.).

Subtracting the femur from the CT scan, the false acetabulum in the ilium displayed a round and deepened articulating surface (Fig. 3.).

\section{DISCUSSION}

A shortened limb with adduction, internal rotation, and flexion of the hip joint is a typical presentation of posterior hip dislocation in trauma patients. However, developmental hip dislocation is a common complication in polio patients, and other various conditions can also cause developmental hip dislocation. If a patient has a history of limping gait since early childhood, congenital or developmental dysplasia of the hip should be suspected and careful radiologic evaluation is important to prevent misdiagnosis $(1,2)$.

\section{CONFLICT OF INTEREST}

No potential conflict of interest relevant to this article was reported.

\section{REFERENCES}

1. Odak S, Mangwani J, Ivory J. Pseudoacetabulum a subtle 
radiological feature of a chronic dislocated total hip replacement. BMJ Case Rep. 2012;2012.

2. Park KS, Seon JK, Nah SY, Yoon TR. Total hip arthroplasty after treatment of pseudojoint infection in a patient with a highly dislocated hip. Case Rep Orthop. 2013;2013:947121. 